\title{
The management of total avulsion of the ureter from both ends: Our experience and literature review
}

\author{
Cuneyd Sevinc ${ }^{1}$, Muhsin Balaban ${ }^{1}$, Orkunt Ozkaptan ${ }^{1}$, Ugur Yucetas ${ }^{2}$, Tahir Karadeniz ${ }^{1}$ \\ ${ }^{1}$ Medicana International Istanbul Hospital, Urology Clinic, Istanbul, Turkey; \\ ${ }^{2}$ Istanbul Training and Research Hospital, Urology Clinic, Istanbul, Turkey.
}

\begin{abstract}
Summary Objective: To evaluate the treatment modalities of total ureteral avulsion and to clarify the risk factors of this serious complication. Methods: This study retrospectively analyzed the data of 3 patients with complete ureteral avulsion during ureteroscopy. Of the three patients, two had distal ureteral complete avulsion, and one total ureteral avulsion on both ends. Ureteroneocystostomy (UNC) was immediately performed after distal ureteral avulsion cases. Ileal ureter substition was performed on the same session after the total ureteral avulsion in both ends. Two of the patients were under chronic use of corticosteroid treatment due to diagnosis of idiopathic trombocytopenic purpura and myastenia gravis and all patients had unsuccesful shockwave litotripsy (SWL) treatment history with at least 1 month period before surgery. Results: The patient who had ileal ureter substitution was followed at 3-month intervals by ultrasonography and renal function tests and she was uneventful after a 2 year follow-up period. The patients treated with UNC were followed up at 3 month interval by ultrasonography and renal function tests. They had normal renal function 1 year after the operation Conclusion: Complete ureteral avulsion is a rare but severe complication. Treatment modality can vary and ileal ureter can be applied succesfully in the total ureter avulsion in both ends when bladder capacity is not enough for a Boari flap. Failed SWL and/or corticosteroid treatment history of patients seems to increase the risk of the ureteral avulsion.
\end{abstract}

KEY WORDS: Avulsion; Lithotripsy; Ureterorenoscopy.

Submitted 5 September 2015; Accepted 6 June 2016

\begin{abstract}
INTRODUCTION
Urolithiasis plays an important role in urinary disease. The treatment for ureteral calculi has evolved within the last decade, due to technological advances. A high success rate depends on advanced instruments, the introduction of the new energy sources and devices, better optical quality, and experience. Ureteroscopy is a common endourological technique in urology. If standard recommendations are followed, the method is safe. However, it may sometimes result in complications such as ureteral false passage, ureteral perforation, bleeding, intussusceptions, and avulsion of the ureter; postoperative complications include infection, fever, urinoma, and strictures. The fre-
\end{abstract}

quency of complications varies between $0.5 \%$ and $10 \%$ in the literature (1-3). Ureteral avulsion is the most serious complication and occurs in 0-0.5\% patients (4).

Treating ureteral avulsion is a challenge. Patient comorbidities, the condition of the kidney, severity of the ureteral damage, and experience of the surgeon can affect the choice of treatment. In this study, we aimed to discuss our experience and possible leading factors of this serious complication in the light of the current literature.

\section{Materials AND Methods}

We retrospectively analyzed three ureteral avulsion cases and treatment modalities at two referral hospitals between January 2008 and October 2014. All patients underwent rigid ureteroscopic procedures for removing ureteral stones, and ureteral avulsion occurred during the retrograde examination of the ureter. Of the three patients ( 1 man, 2 women; age: 28-65 years, one right, two left), the avulsed ureter was repaired during the same session of the ureterorenoscopic surgery. The ureter stones were located in the distal ureter in two patients and in the proximal ureter in one patient. All patients had unsuccessful SWL treatment and at least a 2 week interval before further investigation and treatment were applied. Two patients who have been diagnosed with idiopathic thrombocytopenic purpura and myastenia gravis received chronic corticosteroid treatment.

The patients were evaluated with abdominal X-ray, ultrasonography, intravenous urography (IVP), and/or computerized tomography, and the ureterorenoscopy (URS) operation was planned. Patients were asked not to use antithrombotic or antiaggregant agents at least 1 week before the procedure. Urinalysis and urine culture were examined, and in the case of infection, antibiotic therapy was started according to the culture antibiogram.

The URS operation was performed with a $9.5 \mathrm{~F}$ Wolf semirigid ureteroscope with the insertion of a safety guide-wire in the ureter in the lithotomy position. Fluoroscopy was used in all cases, and balloon dilatation was needed in one case for a narrow ureteral orifice. The guide-wire was not inserted proximal to the stones due to the edematous ureter distal to the calculi in all cases and folded distal on the fluoroscopic view. 
Figure 1.

Avulsed ureter from both ends after releasing it from URS.

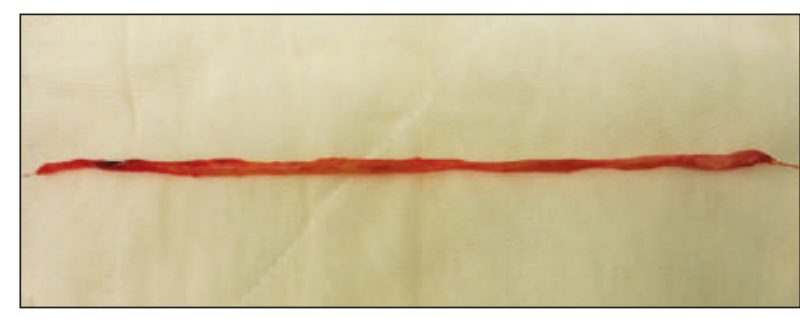

Distal ureteral avulsion occurred during the attempt to reach the distal ureteral stone in one case (diagnosis was made after suspicion of ureteral injury with retrograde ureterography) or when the URS was pulled back with force after the stone was caught with forceps in the other case. Approximately $3 \mathrm{~cm}$ of the avulsed ureter exited from the ureteral orifice coating the stone and forceps. The other avulsed case Repair procedures were performed with an open surgical approach during the same URS session. The bladder was mobilized by freeing its peritoneal attachments. The damaged ureter was identified as it crossed the iliac vessels, mobilized, and divided just above the avulsed segment. The ipsilateral bladder dome was fixed to the psoas muscle, and ureteroneocystostomy (UNC) was performed. A double-J stent was used in both cases.

Total ureteral avulsion in both ends occurred when the ureteroscope was withdrawn after an unsuccessful attempt to remove a proximal ureteral stone and when was planned to switch to a treatment with a flexible URS. The full-length ureteral wall including the proximal, middle, and distal segments were seen covering the scope after the withdrawal of URS (Figure 1). Ileal ureter interposition was performed during the same session of the URS procedure after informed consent was received from the patient's family. The patient's bladder capacity was insufficient for Boari flap ureteroplasty and psoas hitch; thus, ileal interposition was used to reconstruct a
Table 1.

Patients and operation characteristics.

\begin{tabular}{|lccc|}
\hline Case & $\mathbf{1}$ & $\mathbf{2}$ & $\mathbf{3}$ \\
\hline Age (year) & 65 & 38 & 28 \\
\hline Sex & $\mathrm{F}$ & $\mathrm{F}$ & $\mathrm{M}$ \\
\hline Ureteral stone diameter (mm) & 10 & 12 & 13 \\
\hline Side & Right & Left & Left \\
\hline Operation & UNC+Psoas & lleal Ureter & UNC+Psoas \\
& Hitch & substition & Hitch \\
\hline Intervation time & Same session & Same session & Same session \\
\hline Comorbidities & Myastenia & Idiopathic & - \\
& gravis & trombocytopenic & \\
\hline Steroid usage & purpure & \\
\hline SWL treatment before URS & Yes & Yes & No \\
\hline Follow up (month) & 60 & Yes & Yes \\
\hline
\end{tabular}

UNC: Ureteroneocystostomy; SWL: Shockwave lithotripsy URS: Ureterorenoscopy; F: Female; M: Male

new ureter. The intestinal substitute was derived from the terminal ileum measuring $15 \mathrm{~cm}$ in length with preservation of the blood supply. The anastomosis was made between the cephalic end of the ileal tube and the pelvis of the kidney using interrupted 4/0 absorbable sutures with a 6 F double-J stent application.

The nephrostomy tube was also inserted in the same side. The distal end of the ileal tube was then anastomosed to the bladder with the nonrefluxing procedure. The characteristics of the study patients are given in Table 1.

\section{RESULTS}

The postoperative period was uneventful. Ureteral J stent was removed 6 weeks after surgery in all cases.. Followup was performed with renal functional tests and urinary ultrasonography at 3-month periods. Radiological investigation with computed tomography urography was done at the third month. Both renal parenchymas were normal, and there was no hydronephrosis (Figure 2).

Figure 2.

A: $C T$ of the abdomen.

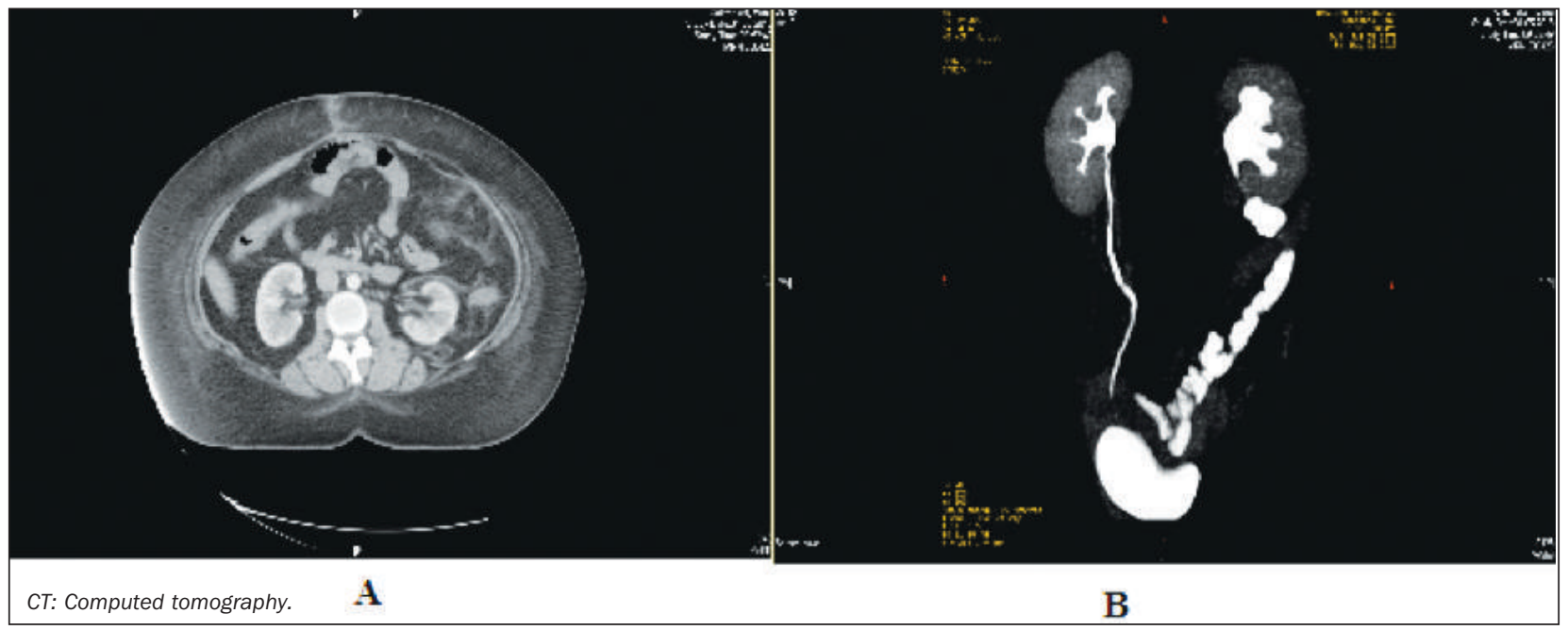


The patients were followed up for 30 months (12-60 months), and during that period, no hydronephrosis or gross hematuria was seen. The ileal ureter substitution case had two episodes of urinary infection and tolerable pain after the surgery until the 3rd month but after then the follow-up was uneventful.

\section{Discussion}

Ureteroscopic examination or treatment procedures may lead to various complications; such as stone residuals, mucosa injury, perforation, bleeding, and edema (5). Postoperative complications such as infection, fever, urinoma, and stricture can also be seen. Ureteral avulsion is a rare but serious complication; fortunately, its incidence is only $0.06 \%$ to $0.45 \%$ (6). Although ureteral avulsion is rare, this catastrophic complication should be taken into consideration while performing an ureteroscopy, and an urologist should be familiar with management options in different avulsion scenarios. The paucity of literature on this complication and its management strategies may lead to urologists in trouble when they plan a repair procedure due to this unexpected rare serious complication. Moreover, comorbidities, the age of the patient, the condition of the ipsilateral and contralateral kidney, location and severity of the ureteral damage, patient expectations, and surgeon experience can affect the choice of the treatment.

Risk factors for ureteral avulsion include symptomatic stones persisting $>3$ months, stones $>5 \mathrm{~mm}$, proximal ureteral distention, stones tightly encapsulated by granulation tissues, and a strong sense of tightness when the ureteroscope is moved. The muscle in the proximal ureteral tissue is weaker, and therefore, the use of a stone basket to remove the impacted stone in the upper third of the ureter increases the risk of avulsion (7). We usually do not use a basket catheter to remove stones or stone fragments. Alternatively, grasping forceps are used to remove stone fragments larger than $3 \mathrm{~mm}$, and sometimes, the ureteroscope is withdrawn to cause fragmented stones to disperse (8). A rigid ureteroscope is also a risk factor for ureteral avulsion as presented in the literature $(9,10)$ and in the three patients in our series. Common features of our patients were symptomatic stone at least 3 months with three SWL sessions before being admitted to our institution for further evaluation and treatment. All stones were larger than $10 \mathrm{~mm}$.

SWL treatment may induce inflammation and increase the fragility of the ureter at the location of the stone. Goktas et al. showed that urine inflammatory cytokines increased after SWL treatment (11). Thus, unsuccessful SWL treatment seems to increase the risk of avulsion. Two patients were on chronic corticosteroid treatment due to a diagnosis of idiopathic thrombocytic purpura and myastenia gravis. Steroid treatment causes muscle atrophy in rat models (12). Chronic use of the corticosteroid treatment may lead to atrophy of the muscle in the ureter wall, and this condition might facilitate the avulsion. Minimal pressure was applied on that patient's ureteroscopy before the stone was reached, and avulsion of the ureterovesical junction (UVJ) and the ureteropelvic junction (UPJ) occurred when the URS was with- drawn to change it with a flexible URS to reach the stone (Figure 1). Moreover, the ureteral region around the stone proximal to the avulsed ureter was seen as inflamed, thinned, and necrosed during the open reconstructive surgery.

Ureteral avulsion is difficult to manage. For proximal ureteral avulsion, end-to-end anastomosis can be a good choice. Boari flap and psoas hitch are recommended for the middle third of ureteral avulsion. For distal ureteral injuries, ureteral re-implantation is recommended (13). If avulsion is diagnosed during the postoperative period, a percutaneous nephrostomy can be placed, and a definitive treatment can be performed when the patient is stable (7). Nephrectomy is also reported as an option for extensive injuries of the ureter in the literature (14). For extensive injuries like one case in our series, there are limited options such as ileal interposition or renal autotransplantation. Appendix interposition has also been reported as a treatment option for extensive injuries in some literature $(15,16)$.

There is a paucity of literature on the surgery approach of the total ureteral avulsion from both ends. Ordon et al. reported three cases that named it as "the scabbard avulsion". A nephrostomy tube was placed postoperatively, and finally, all patients underwent a nephrectomy (14). Ge et al. (17) presented four cases of ureteral avulsion, and two occurred on both ends of the ureter. The Authors reported that one patient was treated with autotransplantation and the other underwent pyeloureterostomy plus greater omentum investment outside. The kidney of the patient who underwent autotransplantation was normal in the follow-up while the other patient underwent nephrectomy after 25 months of follow-up. Thai et al. (18) reported six ureteral avulsion cases in their series. Five had avulsion on the UPJ and the UVJ. The authors preferred nephrectomy for one patient, Boari flap for two patients, ileal interposition for one patient, and ureteral re-implantation for one patient. Unsal et al. (19) reported four ureteral avulsion cases in their series, and they performed two Boari flap and two ureteral re-implantation procedures. One of the ureteral re-implantation cases was normal during the follow-up period while the other case developed hydronephrosis. We had three cases of ureteral avulsion and managed them with ureteroneocystomy with a psoas hitch or ileal ureter substitution (Table 2).

In this study, we presented three ureter avulsion cases with possible risk factors that facilitate this serious complication and management strategies. For distal ureteral avulsion, we performed the UNC with psoas hitch. We did not prefer the Boari flap because after we mobilized the bladder and fixed the dome to the psoas muscle, we obtained enough distance for the UNC procedure.

We applied anti-reflux surgery techniques for both distal ureteral avulsion cases. For total ureteral avulsion in both ends, we prefer ileal ureter substitution due to our experience of orthotopic ileal bladder diversion for invasive bladder cancer. Despite the common belief that this surgery is a complex procedure and has potential complications such as anastomotic stenosis, ileus, and urinary obstruction.

The postoperative period was uneventful except for one 
Table 2.

Series of ureteral avulsion form both ends in the literature and in our own series.

\begin{tabular}{|c|c|c|c|c|}
\hline Literature & Case number & Reason & Treatment & Kidney in follow-up \\
\hline Ordon et al. (14) & 3 & Ureteroscope & 3 Nephrectomy & - \\
\hline Ge et al. (17) & 2 & NA & $\begin{array}{c}1 \text { Autotransplatation } \\
1 \text { Pyeloureterostomy plus greater } \\
\text { omentum investment outside }\end{array}$ & $\begin{array}{c}\text { Normal } \\
\text { Underwent nephrectomy }\end{array}$ \\
\hline Taie et al. (18) & 5 & Ureteroscope & $\begin{array}{l}1 \text { Ureteral re-implant } \\
2 \text { Boari flap } \\
1 \text { Ileal interposition } \\
1 \text { Nephrectomy }\end{array}$ & $\begin{array}{l}\text { Underwent nephrectomy } \\
\text { Both normal } \\
\text { Normal } \\
-\end{array}$ \\
\hline Unsal et al. (19) & 4 & Ureteroscope & $\begin{array}{l}2 \text { Ureteral re-implant } \\
2 \text { Boari flap }\end{array}$ & $\begin{array}{c}1 \text { normal, } 1 \text { hydronephrosis } \\
\text { Both normal }\end{array}$ \\
\hline Our series & 3 & Ureteroscope & $\begin{array}{l}2 \text { Ureteral re-implant } \\
1 \text { Ileal interposition }\end{array}$ & $\begin{array}{l}\text { Both normal } \\
\text { Normal }\end{array}$ \\
\hline
\end{tabular}

NA: Not applicable.

attack of urinary infection. For this case, autotransplantation or ureteral re-implantation is an alternative approach, but due to the paucity of literature on ureteral re-implantation, this approach was not preferred. In addition, since we have ileal bladder experience, we did not prefer kidney autotransplantation.

\section{Conclusions}

Ureteral avulsion is a rare but very serious complication of the URS procedure. UNC is an option for distal ureteral complete avulsion cases and ileal ureteral substitution is should be kept in mind for total avulsion of the ureter in both ends. In the case of SWL and/or corticosteroid treatment history of patients with URS, the risk of ureteral avulsion seems to increase, and more care should be taken during the procedure.

\section{REFERENCES}

1. Al-Awadi K, Kehinde EO, Al-Hunayan A, et al. Iatrogenic ureteric injuries: incidence, aetiological factors and the effect of early management on subsequent outcome. Int Urol Nephrol. 2005; 37:235-241.

2. Butler MR, Power RE, Thornhill JA, et al. An audit of 2273 ureteroscopies-a focus on intra-operative complications to justify proactive management of ureteric calculi. Surgeon. 2004; 2:42-46.

3. Fuganti PE, Pires S, Branco R, et al. Predictive factors for intraoperative complications in semirigid ureteroscopy: analysis of 1235 ballistic ureterolithotripsies.Urology. 2008; 72:770-774.

4. D'Addessi A, Bassi P. Ureterorenoscopy: avoiding and managing the complications. Urol Int. 2011; 87:251-259.

5. Abdelrahim AF, Abdelmaguid A, Abuzeid $H$, et al. Rigid ureteroscopy for ureteral stones: factors associated with intraoperative adverse events. J Endourol. 2008; 22:277-280.

6. Ben Slama MR, Zaafrani R, Ben Mouelli S, et al. Ureterocalicostomy: last resort in the treatment of certain forms of ureteropelvic junction stenosis. Report of 5 cases. Prog Urol. 2005; 15:646-649.

7. de la Rosette JJ, Skrekas T, Segura JW. Handling and prevention of complications in stone basketing. Eur Urol. 2006; 50:991-998.

8. Canguven O, Boz M, Bulbul M, et al. Withdraw of the ureteroscope causes fragmented ureter stones to disperse. Int Braz J Urol. 2013; 39:756-7.
9. Martin X, Ndoye A, Konan PG, et al. Apropos of 4 cases of avulsion of the ureter. Prog Urol. 1998; 8:358-362.

10. Alapont JM, Broseta E, Oliver F, et al. Ureteral avulsion as a complication of ureteroscopy. Int Braz J Urol. 2003; 29:18:22.

11. Goktas C, Coskun A, Bicik Z, et al. Evaluating ESWL-induced renal injury based on urinary TNF-alpha, IL-1alpha, and IL-6 levels. Urol Res. 2012; 40:569-73.

12. Ma K, Mallidis C, Bhasin S, et al. Glucocorticoid-induced skeletal muscle atrophy is associated with upregulation of myostatin gene expression. Am J Physiol Endocrinol Metab. 2003; 285:363-71.

13. Gupta V, Sadasukhi TC, Sharma KK, et al. Complete ureteral avulsion. ScientificWorld Journal. 2005; 28:125-127.

14. Ordon M, Schuler TD, Honey RJ. Ureteral avulsion during contemporary ureteroscopic stone management: "the scabbard avulsion". J Endourol. 2011; 25:1259-1262.

15. Dagash H, Sen S, Chacko J, et al. The appendix as ureteral substitute: a report of 10 cases. J Pediatr Urol. 2008; 4:14-19.

16. Juma S, Nickel JC. Appendix interposition of the ureter. J Urol. 1990; 144:130-131.

17. Ge C, Li Q, Wang L, et al. Management of complete ureteral avulsion and literature review. a report on four cases. J Endourol. 2011; 25:323-326.

18. Taie K, Jasemi M, Khazaeli D, et al. Prevelance and management of complications of ureteroscopy: a seven-year experience with introduction of a new maneuver to prevent ureteral avulsion. Urol J. 2012; 9:356-360.

19. Unsal A, Oguz U, Tuncel A, et al. How to manage total avulsion of the ureter from both ends: our experience and literature review. Int Urol Nephrol. 2013; 45:1553-60.

\section{Correspondence \\ Cuneyd Sevinc, MD \\ cuneydsevinc@yahoo.com \\ Muhsin Balaban, MD (Corresponding Author) muhsinbalaban1980@yahoo.com \\ Orkunt Ozkaptan, MD \\ Tahir Karadeniz, MD}

Medicana International Istanbul Hospital, Urology Clinic, Yeni mah., Pegagaz Sok. Soyak Evreka:A5-44, Soganlik, Kartal 34880 Istanbul, Turkey

Ugur Yucetas, MD dryucetas@yahoo.com

Istanbul Training and Research Hospital, Urology Clinic, Istanbul, Turkey 